Draft VERSion November 14, 2018

Preprint typeset using $\mathrm{LAT}_{\mathrm{E}} \mathrm{X}$ style emulateapj v. 04/17/13

\title{
STELLAR PARAMETERS AND ACCRETION RATE OF THE TRANSITION DISK STAR HD 142527 FROM X-SHOOTER.
}

\author{
I. Mendigutía \\ School of Physics \& Astronomy, University of Leeds, Woodhouse Lane, Leeds LS2 9JT, UK \\ J. FAIRLAMB \\ School of Physics \& Astronomy, University of Leeds, Woodhouse Lane, Leeds LS2 9JT, UK \\ B. Montesinos \\ Centro de Astrobiología, Departamento de Astrofísica (CSIC-INTA), ESAC Campus, P.O. Box 78, 28691 Villanueva de la Cañada,
Madrid, Spain. \\ R.D. OudmaijeR \\ School of Physics \& Astronomy, University of Leeds, Woodhouse Lane, Leeds LS2 9JT, UK \\ J.R. NAJITA \\ National Optical Astronomy Observatory, 950 N. Cherry Avenue, Tucson, AZ 85719, USA \\ S.D. BRITTAIN \\ Department of Physics and Astronomy, Clemson University, Clemson, SC 29634-0978, USA
}

M.E. VAN DEN ANCKER

European Southern Observatory, Karl-Schwarzschild-Str. 2, D-85748 Garching b. München, Germany

Draft version November 14, 2018

\begin{abstract}
HD 142527 is a young pre-main sequence star with properties indicative of the presence of a giant planet or/and a low-mass stellar companion. We have analyzed an X-Shooter/Very Large Telescope spectrum to provide accurate stellar parameters and accretion rate. The analysis of the spectrum, together with constraints provided by the SED fitting, the distance to the star $(140 \pm 20 \mathrm{pc})$ and the use of evolutionary tracks and isochrones, lead to the following set of parameters $T_{\text {eff }}=6550 \pm$ $100 \mathrm{~K}, \log g=3.75 \pm 0.10, L_{*} / L_{\odot}=16.3 \pm 4.5, M_{*} / M_{\odot}=2.0 \pm 0.3$ and an age of $5.0 \pm 1.5 \mathrm{Myr}$. This stellar age provides further constrains to the mass of the possible companion estimated by Biller et al. (2012), being in-between 0.20 and $0.35 \mathrm{M}_{\odot}$. Stellar accretion rates obtained from UV Balmer excess modelling, optical photospheric line veiling, and from the correlations with several emission lines spanning from the UV to the near-IR, are consistent to each other. The mean value from all previous tracers is $2( \pm 1) \times 10^{-7} \mathrm{M}_{\odot} \mathrm{yr}^{-1}$, which is within the upper limit gas flow rate from the outer to the inner disk recently provided by Cassasus et al. (2013). This suggests that almost all gas transferred between both components of the disk is not trapped by the possible planet(s) in-between but fall onto the central star, although it is discussed how the gap flow rate could be larger than previously suggested. In addition, we provide evidence showing that the stellar accretion rate of HD 142527 has increased by a factor $\sim 7$ on a timescale of $2-5$ years.

Keywords: Accretion, accretion disks - circumstellar matter — planet-disk interactions - protoplanetary disks — stars: fundamental parameters — stars: pre-main sequence
\end{abstract}

\section{INTRODUCTION}

Since the first discovery of a planet orbiting a star other than the Sun (Mayor \& Queloz 1995), around 1000 exoplanets have been confirmed around main sequence stars with different properties. Indeed, the latest results from Kepler are consistent with nearly every Sun-like star in the galaxy harboring a planet (Fressin et al. 2013).

I.Mendigutia@leeds.ac.uk
Planet formation is expected to occur in the gas and dust disks that surround young stars during the first $~$ 10 Myr. However, the detection of planets caught in their process of formation is limited only to a few candidates (e.g. Huélamo et al. 2011; Kraus \& Ireland 2012; van Eyken et al. 2012; Brittain et al. 2013). Nevertheless, several pre-main sequence (PMS) stars present specific properties that make them the ideal candidates to look for planets in their disks. 
One of these objects is the isolated, intermediatemass PMS star HD 142527, which shows several observational signatures of ongoing gas giant planet formation. Using spectra taken by the SWS and LWS instruments mounted on the Infrared Space Observatory, Malfait et al. (1999) reported two different dust components in its almost face-on disk. This double nature is accompanied by a specific morphology. Near and midinfrared images show that the disk has a large inner hole, two bright facing arcs, and a spiral arm extending from one of them (Fukagawa et al. 2006; Fuijwara et al. 2006). Verhoeff et al. (2011) provided a more precise description of the circumstellar environment of HD 142527, constituted by a geometrically thin inner disk that extends from 0.3 to $30 \mathrm{AU}$, an optically thin halo-like component of dust in the inner disk regions, and a massive outer disk running from 130 AU up to 200 AU. These authors find that the peculiar properties of this system are indicative of on-going planet formation (see also Rameau et al. 2012). Casassus et al. (2012) proposed that the gap between the inner and outer disk could have been carved out by a giant planet located at around $\sim 90$ $\mathrm{AU}$, which causes perturbations in the whole disk and the appearance of spiral arms. More recently, Casassus et al. (2013) used ALMA observations to report the presence of planet-induced CO gas channels flowing from the outer to the inner disk at a rate ranging between $7 \times 10^{-9}$ and $2 \times 10^{-7} \mathrm{M}_{\odot} \mathrm{yr}^{-1}$. This flow would supply material to the inner disk and is consistent with a previous estimate of the stellar accretion rate (Garcia Lopez et al. 2006, see below). Other possible scenarios explaining the peculiar properties of the HD 142527 include the possible presence of a stellar companion (Fukagawa et al. 2006; Baines et al. 2006; Biller et al. 2012; Close et al. 2014).

While observations of HD 142527 have generated a great deal of excitement, the stellar and accretion properties are poorly characterized. Based on unpublished spectra and photometry, HD 142527 was classified as a Herbig Ae/Be (HAeBe) star by Waelkens et al. (1996), and a spectral type F6-F7III is the most commonly assumed (Houk 1978; van den Ancker et al. 1998). From an Hipparcos distance of $\sim 200 \mathrm{pc}$, van den Ancker et al. (1998) derived a stellar mass and luminosity of $3.5 \mathrm{M}_{\odot}$ and $69 \mathrm{~L}_{\odot}$, a surface temperature of $6300 \mathrm{~K}$ and an age of $10^{5}$ years. These values are partially similar to the ones obtained in subsequent works van Boekel et al. 2005; Manoj et al. 2006). In contrast, Fukagawa et al. (2006) assumed a distance of $140 \mathrm{pc}$, stellar mass of $1.9 \mathrm{M}_{\odot}$, and an age of 2 Myr. Recent works (e.g. Biller et al. 2012; Rameau et al. 2012) assume the parameters provided by Verhoeff et al. (2011): $\mathrm{d}=145 \mathrm{pc}, \mathrm{M}=2.2 \mathrm{M}_{\odot}, \mathrm{L}=20$ $\mathrm{L}_{\odot}, \mathrm{T}=6250 \mathrm{~K}$, age $=5 \mathrm{Myr}, \mathrm{R}=3.8 \mathrm{R}_{\odot}$. Regarding the stellar accretion rate, there are only two estimates in the literature. Garcia Lopez et al. (2006) provided $\sim 7$ $\times 10^{-8} \mathrm{M}_{\odot} \mathrm{yr}^{-1}$ from the correlation with the Br $\gamma$ luminosity in Calvet et al. (2004). More recently, Salyk et al. (2013) obtained a correlation between the accretion luminosity derived from different observational methods, and the $\mathrm{H}$ I Pfund $\beta$ line luminosity. Using this correlation, a mass accretion rate of $\sim 1 \times 10^{-7} \mathrm{M}_{\odot} \mathrm{yr}^{-1}$ was derived for HD 142527. However, accretion rates obtained from individual lines suffer from large uncertainties, while the average derived from several line luminosities measured simultaneousy is a more robust estimate (Rigliaco et al. 2012). Moreover, accretion variability has been reported for several HAeBes (Mendigutía et al. 2011, 2013), for which estimates obtained at different epochs become necessary.

The parameters characterizing the central star determine the energy received by the disk and influence its geometry, energy balance, accretion rate and contribution to the spectral energy distribution. It is our aim to provide an independent, self-consistent study of the stellar parameters of HD 142527, that will serve for future detailed analysis of its disk properties. We make use of an X-Shooter/Very Large Telescope (VLT; Vernet et al. 2011) spectrum, whose wide wavelength coverage from the UV to the near-IR provides an accurate estimate of the mass accretion rate from several tracers observed simultaneously.

This paper is organized as follows. Section 2 describes the X-Shooter spectrum used in this work. The stellar properties of HD 142527 are derived in Sect. 3, and the accretion rate in Sect. 4. Results are summarized and discussed in Sect. 5

\section{OBSERVATIONAL DATA}

This work is mainly based on the spectrum of HD 142527 taken on 2010-04-01 UT08:43, using the XShooter spectrograph mounted on the VLT (Vernet et al. 2011). X-Shooter covers a wide spectral range $(300 \mathrm{~nm}-$ $2.5 \mu \mathrm{m})$ across three arms: $300-590 \mathrm{~nm}, 530 \mathrm{~nm}-1.0 \mu \mathrm{m}$, and 1.0-2.4 $\mu \mathrm{m}$ with the UVB, VIS and NIR arms, respectively. The smallest slit widths available were used: 0.5" (UVB), 0.4" (VIS), and 0.4" (NIR). This gives the highest spectral resolution achieveable with X-Shooter; $\mathrm{R}=9100,17400$ and 11300 , respectively. The target was observed in nodding mode with a total exposure time of 82 seconds.

The spectrum was reduced in two stages. The first follows the standard reduction from the X-Shooter pipeline v0.9.7 (Modigliani et al. 2010). The pipeline-reduced 1D spectrum is suitable for measurement of spectral lines but not for an analysis of the continuum shape, due to problems encountered when the separate echelle orders are merged. Therefore, a second stage of reduction was required to derive the proper SED shape. The flux standard observed on the same night has a poor $\mathrm{S} / \mathrm{N}$ ratio and is rejected in favour of a higher $\mathrm{S} / \mathrm{N}$ telluric standard, observed shortly after the target star. The exact flux of the telluric standard is not known and therefore cannot produce an accurate flux correction. However, absolute flux calibration is not required for this work, which only needs from an accurate determination of the SED shape in the UVB arm (Sect. 4.1). In order to perform this correction, each echelle order of the telluric standard was corrected individually by comparing against a model of the same spectral type. We obtained accurate stellar parameters for our telluric standard, HIP 77968, by comparing the wings of the Balmer re-combination lines with a grid of Kurucz-Castelli model atmospheres from Munari et al. (2005). The Balmer wings have been shown to be sensitive to change in both $\mathrm{T}_{\text {eff }}$ and $\log \mathrm{g}$ (Montesinos et al. 2009). Using this method, we derived $\mathrm{T}_{\text {eff }}=12000 \pm 250 \mathrm{~K}$ and $\log \mathrm{g}=4.2 \pm 0.15$ for the telluric star. These values agree within the spectral type range of the literature (e.g. Straizys \& Kuriliene 1981), 
and were used for the corrections. Each echelle order of the telluric spectrum was divided through by the corresponding Kurucz model atmosphere to produce a correction function. This was then applyed to the 1D echelle orders of HD 142527, resulting in each echelle order now having the correct SED shape. They were then merged together where they overlap to produce the corrected 1D spectra.

\section{STELLAR PARAMETERS}

The problem of the distance to this star is fundamental to determine the stellar parameters, which in turn effects the accretion rate estimates (Sect. 4). The parallax from the Hipparcos catalogue is $\pi=5.04 \pm 1.18$ mas, which implies a distance of $198.4_{-37.6}^{+60.7} \mathrm{pc}$; van Leeuwen (2007) gives a smaller value for the parallax, namely $\pi=4.29 \pm 0.98$ mas $\left(\mathrm{d}=233.1_{-43.4}^{+69.9} \mathrm{pc}\right)$. However, in both cases $\sigma_{\pi} / \pi>0.17$, therefore the Lutz-Kelker bias (Lutz \& Kelker 1973) prevents from deducing a reliable distance to the star from the parallaxes. The consensus is to adopt a shorter distance of $140( \pm 20)$ pc (Fukagawa et al. 2006), which is the one adopted in this work. There are strong reasons supporting that assumption: the star is within $\sim 1$ degree of the Lupus IV cloud (at a mean distance of $140 \mathrm{pc}$ ) and has known Lupus and Sco-Cen members in its vicinity (the de Zeeuw et al. 1999, catalog shows four Sco-Cen members within 2 degrees, and nine within 3 degrees). In addition, the proper motion and radial velocity of HD 142527 are similar to those of other of stars in the Lupus clouds (see van Leeuwen 2007; Galli et al. 2013).

A visual inspection of the blue part of the X-Shooter spectrum and a comparison with MK standars (see e.g. Gray \& Corbally 2009) hints that the spectral type is between $\sim \mathrm{F} 3$ and F8, whereas the luminosity class is between V and III. However, the photospheric lines that are primary indicators for the stellar temperature in F stars, namely the Balmer hydrogen lines, cannot be used here since their profiles show a non-photospheric component caused by accretion (Sect. 4.3). For the same reason, the $\mathrm{Ca}$ II HK and Na ID lines, as well as Ca I at $4226 \AA$ and Fe I at $4046,4383 \AA$ (which are also temperature indicators in combination with $\mathrm{H} \gamma$ and $\mathrm{H} \delta$; Gray \& Corbally 2009), cannot be used either. In addition, the spectral resolution -lower than that of a echelle spectrum- prevents from applying diagnostics based purely on line ratios. Therefore an alternative was followed to further constrain the stellar parameters.

We have used the region $4200-4400 \AA$ to obtain an estimate of the effective temperature (Fig. 1). A grid of high-resolution spectra with $T_{\text {eff }}=6300-6700 \mathrm{~K}$, in steps of $100 \mathrm{~K}$, and $\log g=3.0,3.5,4.0$, 4.5 was synthetized. The programmes SYNTHE and ATLAS (Kurucz, 1993) fed with the models describing the stratification of the stellar atmospheres (Castelli \& Kurucz 2003) and solar abundances were used. For a given gravity, the depth/equivalent width of the vast majority of the lines decrease as the temperature increases -as expected- so we have used this fact to constrain the value of $T_{\text {eff }}$. To quantify that, we normalized to unity the observed spectrum and the synthetic models, and integrated the area below the spectral lines (avoiding the $\mathrm{CH}$ G-band around $4300 \AA$, which shows clear gravity effects for a given tem-

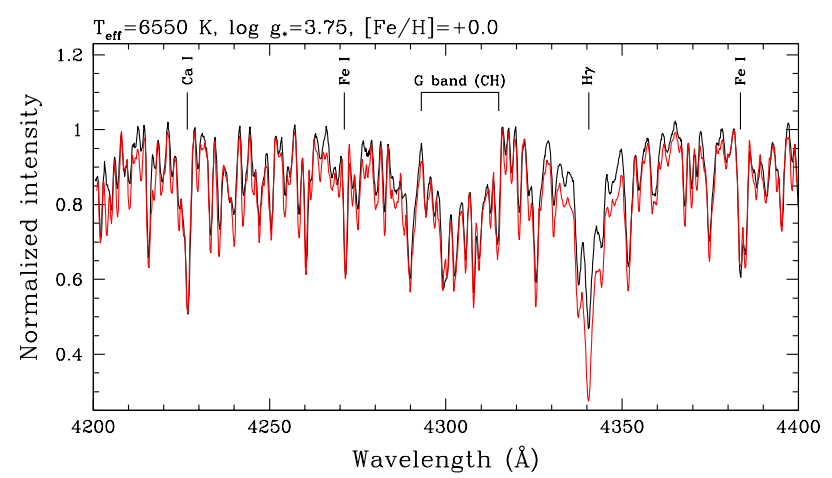

Figure 1. Observed spectrum of HD 142527 (black) in the interval 4200-4400 ^ and best fit synthetic spectrum (red, see text) computed with $\mathrm{T}_{\text {eff }}=6550 \mathrm{~K}, \log g_{*}=3.75$, solar metallicity and broadened with $v \sin i=45 \mathrm{~km} / \mathrm{s}$ (Glebocki \& Gnacinsky 2005).

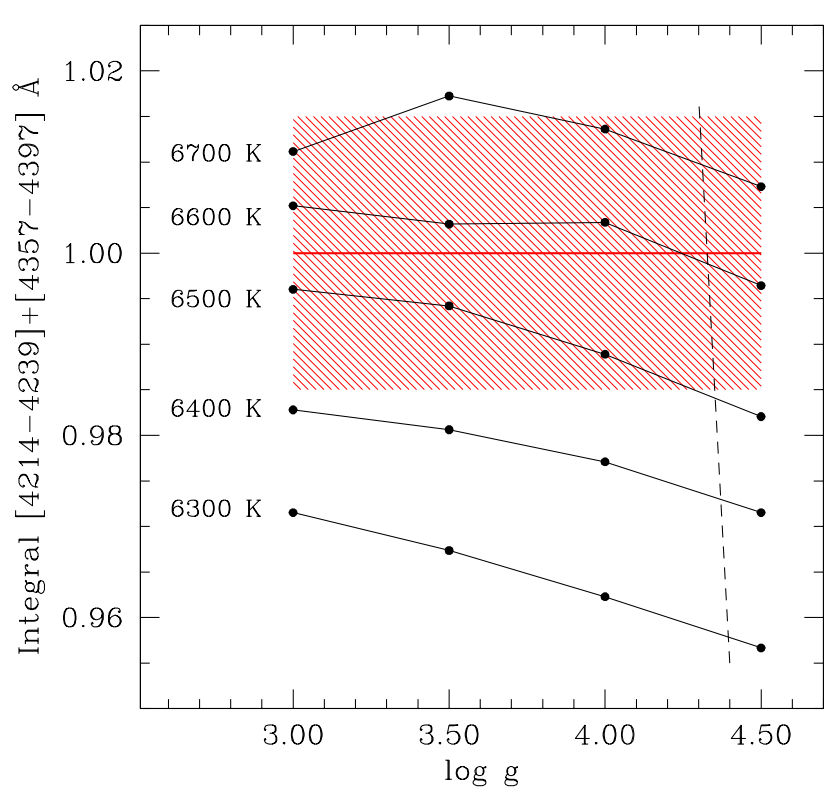

Figure 2. Integrals of the area under the normalized intensity of the synthetic models (black dots and lines) in the regions [4214$4239]+[4357-4397] \AA$ divided by the area under the intensity of the observed spectrum in the same region (thick red line), that has been scaled to 1.0. The shaded region marks the uncertainty in the latter integral due to potencial inaccuracies in the normalization of the observed spectrum. The dashed black line marks the values of $\log g$ for main-sequence stars. See text for details

perature, and $\mathrm{H} \gamma$ ). This analysis has the advantage that the integrals do not depend on the projected rotational velocity $v \sin i$, because rotational broadening conserves equivalent widths. The results can be found in Fig. 2 The thick horizontal red line marks the value of the area contained under the observed spectrum, that has been scaled to 1.0, and the shaded area accounts for potential inaccuracies in the normalization of the observed spectrum and hence in the value of the integral; the black dots and lines joining them are the areas contained under the corresponding synthetic models normalized to the area under the observed spectrum. The dashed line marks the values of $\log g$ for main-sequence stars in this range of temperatures. As it can be seen, the effective temperature should be higher than $6400 \mathrm{~K}$ but not than 6700 K. 
Table 1

Stellar parameters of HD 142527 derived in this work

\begin{tabular}{lll}
\hline \hline Effective temperature & $T_{\text {eff }}(\mathrm{K})$ & $6550 \pm 100$ \\
Stellar mass & $M_{*}\left(\mathrm{M}_{\odot}\right)$ & $2.0 \pm 0.3$ \\
Surface gravity & $\log g_{*}\left(\mathrm{~cm} / \mathrm{s}^{2}\right)$ & $3.75 \pm 0.10$ \\
Stellar luminosity & $L_{*}\left(\mathrm{~L}_{\odot}\right)$ & $16.3 \pm 4.5$ \\
Age & $t(\mathrm{Myr})$ & $5.0 \pm 1.5$ \\
Colour excess & $E(B-V)(\mathrm{mag})$ & $0.25 \pm 0.04$ \\
\hline
\end{tabular}

In order to constrain the value of $\log g$, all the luminosity criteria given by Gray \& Corbally (2009) for F-type stars have been checked for the latter range of temperatures. Values between $\log g=3.5$ and 4.0 are valid but unfortunately none of the criteria provide clues to break the degeneracy and give an accurate value for the gravity. This problem was circunvented using the distance to the star. We have taken as a baseline the model with $T_{\text {eff }}=6550 \mathrm{~K}$ and $\log g=3.75$ (right in between the limits in temperature and gravity given by the spectral analysis above). A Kurucz low-resolution model with these parameters was fitted to the available optical photometry (Johnson $B V$ and Cousins $I_{\mathrm{c}}$ photometry from the Hipparcos CDS catalogue I/239/hip_main!1). By integrating the dereddened fit, the observed photospheric flux can be estimated. On the other hand, using evolutionary tracks and isochrones (Fig. 3), the point $(6550 \mathrm{~K}$, $3.75)$ can be taken from the $\left(T_{\text {eff }}, \log g\right)$ HR diagram to the $\left(T_{\text {eff }}, L_{*} / L_{\odot}\right)$ HR diagram, allowing us to estimate the stellar luminosity. From the photospheric flux and the luminosity, the distance can be estimated; for that particular model it turns out that $d=144 \mathrm{pc}$, in excellent agreement with the distance of $140 \mathrm{pc}$ assumed for the star. An uncertainty of $\pm 20 \mathrm{pc}$, translated into the luminosities, implies an uncertainty of \pm 0.10 in $\log g$. Distances derived for values of $\log g$ outside the range $3.75 \pm 0.10$ are inconsistent with the star being at $140 \pm 20$ pc.

The uncertainty in the temperature, set in $\pm 150 \mathrm{~K}$ by the results displayed in Fig. 2, can be further reduced to $\pm 100 \mathrm{~K}$ when models with temperatures outside the range $6550 \pm 100 \mathrm{~K}$ are compared with the observed spectrum in the region of the CH G-band (Fig. 1), which for a given $T_{\text {eff }}$ shows a strong dependence with $\log g$. For $T_{\text {eff }}<6450$ and $T_{\text {eff }}>6650 \mathrm{~K}$ the gravities needed to reproduce that feature are incompatible with the star being at $140 \pm 20 \mathrm{pc}$.

Table 1 summarizes the stellar parameters derived for HD 142527 from a combined analysis of the spectrum, SED fitting, and the use of evolutionary tracks and isochrones. These allowed us to also estimate the mass and age of the star. The colour excess in the table corresponds to the reddening required to fit the Kurucz lowresolution spectrum with $6550 \mathrm{~K}, \log g=3.75$ to the observed photometry.

\section{ACCRETION RATE}

Based on the previously obtained stellar parameters, HD 142527 is a giant star with a mass typical of a late type HAeBe star and a temperature more akin to a classical $\mathrm{T}$ Tauri. In order to es-

\footnotetext{
1 from the comparison with a different set of optical measurements (Malfait et al. 1998), there is no evidence for strong photometric variability in HD 142527.
}

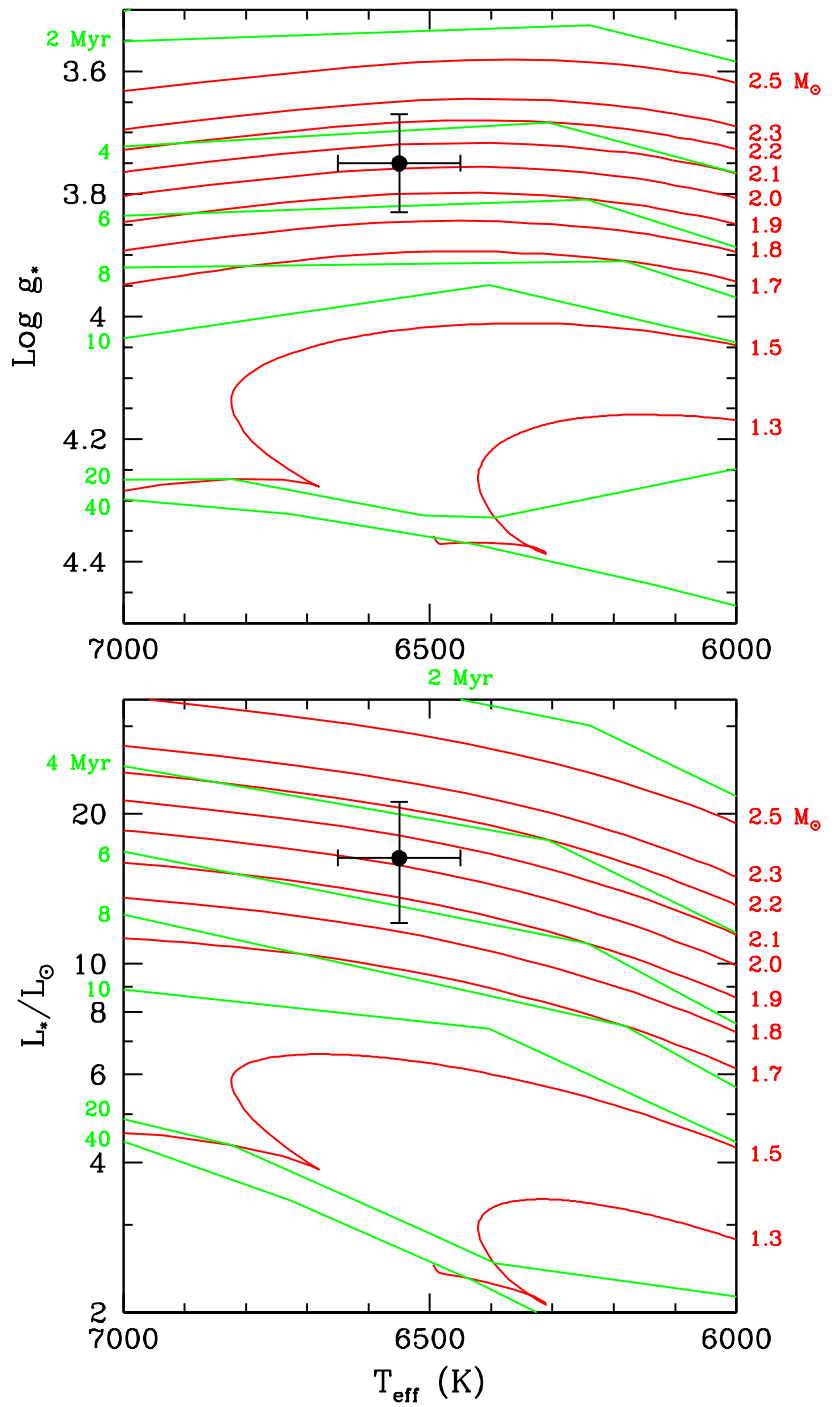

Figure 3. $\log g_{*}-T_{\text {eff }}$ (top) and $L_{*} / L_{\odot}-T_{\text {eff }}$ (bottom) HR diagrams showing the position of HD 142527 according to the set of parameters determined in this work. The Yonsei-Yale set Yi et al. (2001) of tracks (red) and isochrones (green) for solar metallicity have been used.

timate its mass accretion rate $\left(\dot{M}_{a c c}\right)$, it is assumed that material in the disc is transported to the star in the context of magnetospheric accretion (MA hereafter; Uchida \& Shibata 1985; Koenigl 1991; Shu et al. 1994; Bouvier et al. 2007), as this has been shown to hold for intermediate-mass $\mathrm{T}$ Tauris and HAe stars (Calvet et al. 2004; Vink et al. 2002; Muzerolle et al. 2004; Mottram et al. 2007; Donehew \& Brittain 2011; Mendigutía et al. 2011). According to MA, the infalling material shocks the photosphere heating the region and releasing soft X-rays. The majority of these go on to thermalize the surrounding, resulting in emission that is predominately in the UV and optical. This energy is reflected by an excess emission in the Balmer region of the spectrum (Sect. 4.1) or by veiled photospheric lines in the optical region (Sect. 4.2). In addition, accretion estimates based on the two previous observables were found to correlate with the luminosity of several emission lines that span from the UV to the near-IR (Sect. 4.3). 


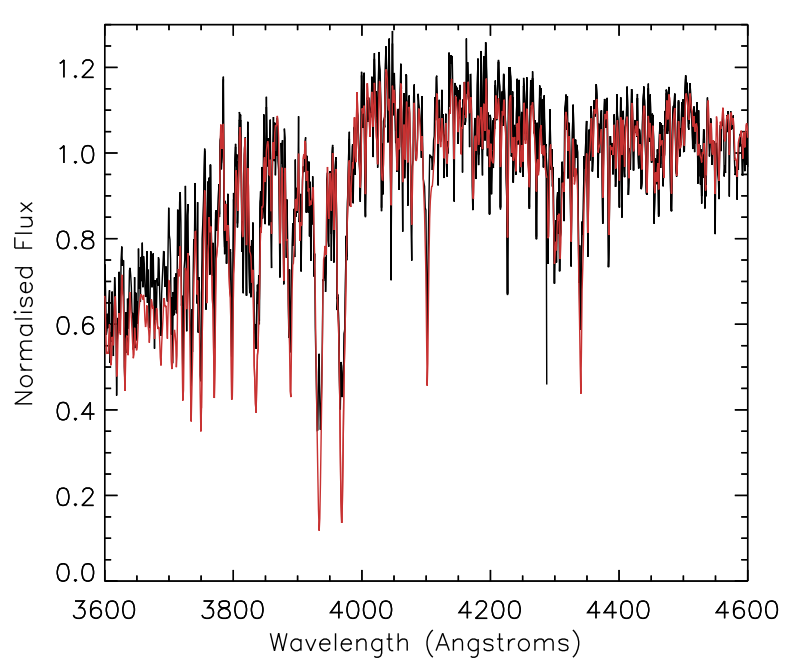

Figure 4. Observed and Kurucz spectra of HD142527 are shown with in black and red, respectively. Both spectra were normalised to 1 at $4000 \AA$ and the observed spectrum was adjusted to match the slope of the model spectrum between 4000-4600. .

\subsection{Balmer Excess}

The Balmer Excess $\left(\Delta D_{B}\right)$ is given by:

$$
\Delta D_{B}=(U-B)_{0}-(U-B)_{\text {dered }}
$$

where $(U-B)_{0}$ is the intrinsic colour of the photosphere and $(U-B)_{\text {dered }}$ is the deredenned, observed colour. To perform a reddening correction the observed spectrum and Kurucz model representing the photosphere are first both normalised to 400nm. From here the slope of the observed spectrum is adjusted to match the spectra of the model between $400-460 \mathrm{~nm}$. This is done by measuring the difference between the observed and model and fitting the points with the Cardelli et al. (1989) extinction law, which is then extrapolated to $360 \mathrm{~nm}$. By applying this fit to the observed spectra the slopes now match and the $\mathrm{U}$ band point for measurement is in the correct place. Due to the slope normalisation the B band is now the same for both object and model (see Fig. 4), and the equation for the Balmer Excess simplifies to:

$$
\Delta D_{B}=2.5 \log \left(\frac{F_{i n t}+F_{a c c}}{F_{*}}\right),
$$

where $F_{\text {int }}$ is the intrinsic flux of the star at $360 \mathrm{~nm}$ (the model in this case), and $F_{a c c}$ is the flux due to accretion at $360 \mathrm{~nm}$, such that $F_{\text {int }}+F_{a c c}$ is the observed flux. This is the expresion used in Donehew \& Brittain (2011). In their approach $\Delta D_{B}$ can be measured without knowledge of a precise flux, and only the SED shape is required. This is appropriate for the characteristics of our X-Shooter spectrum of HD 142527 (see Sect. 2).

The modelling used to determine $\dot{M}_{a c c}$ from $\Delta D_{B}$ closely follows the work of Calvet \& Gullbring (1998), who applyed shock modelling in the context of MA, and the work of Muzerolle et al. (2004) and Mendigutía et al. (2011), who applied the same model to HAeBe stars. We follow the same nomenclature and methodology described in the previous works. For a given set of stellar parameters there is only one observable $\Delta D_{B}$ for each possible value of $\dot{M}_{a c c}$ (Mendigutía et al. 2011). From this, a measurement of $\Delta D_{B}$ can be used to determine the accretion rate. We obtain $\Delta D_{B}=0.12 \pm 0.03$. The excess emission coming from the hot spot of the accretion column can be approximated as a blackbody function: $F^{\mathrm{col}}=\sigma T_{\mathrm{col}}^{4}$, where $T_{\text {col }}$ is the temperature of the hot spot region and $\sigma$ is the Stefan-Boltzmann constant. The total flux from the undisturbed photosphere can also be represented as a blackbody function: $F^{\text {phot }}=\sigma T_{\mathrm{eff}}^{4}$. With these approximations the column temperature can be described as $T_{\text {col }}=\left(\mathcal{F} / \sigma+T_{\text {eff }}^{4}\right)^{1 / 4}$, where $\mathcal{F}$ is the inward flux of energy from the accretion column, adopted to be $10^{12} \mathrm{ergs}^{\mathrm{cm}} \mathrm{cm}^{-2} \mathrm{~s}^{-1}$ as this has been shown to provide appropriate filling factors $(f<0.1)$ for HAeBe objects (Muzerolle et al. 2004; Mendigutía et al. 2011). For the surface temperature of HD 142527 and $\mathcal{F}=10^{12}$ ergs cm $\mathrm{cm}^{-2} \mathrm{~s}^{-1}$, the column temperature is $T_{\text {col }}=11800 \pm 120 \mathrm{~K}$. This corresponds to a filling factor of $f=0.010 \pm 0.004$, which is within the expected range, and an accretion rate of $\dot{M}_{a c c}=1.3( \pm 0.5) \times 10^{-7} \mathrm{M}_{\odot} \mathrm{yr}^{-1}$.

\subsection{Photospheric line veiling}

A second, rough estimate of the accretion rate was obtained from veiled photospheric lines, i.e. those showing weaker absorptions than the corresponding naked photoshere, due to the contribution of the accretion emission. Veiling is estimated by measuring the parameter $r$ $=\left(\mathrm{EW}^{\text {phot }}-\mathrm{EW}\right) / \mathrm{EW}$ on several photospheric absorption lines, where EW is the observed equivalent width, and $\mathrm{EW}^{\text {phot }}$ the photospheric equivalent width estimated from a Kuruckz synthetic spectrum with the same stellar parameters as HD 142527. Figure 5]shows excerpts of the $\mathrm{X}$-Shooter spectrum in the range $583-653 \mathrm{~nm}$ (i.e., the range roughly covered by the Johnson $\mathrm{R}$ photometric filter), where we focused our measurements. Veiling is apparent in some photospheric lines, although it is not very intense, with an average value $r=0.3 \pm 0.1$. The uncertainty comes from the propagation of the individual EWmeasurements, which are in turn estimated from those lines with non-detected veiling, with $\mathrm{r}$ values scattering about zero. The excess flux in the $\mathrm{R}$ photometric band is $\mathrm{r} /(1+\mathrm{r})$ times the luminosity of the photosphere in the same band (White \& Basri 2003; White \& Hillenbrand 2004), which is obtained by convolving this photometric filter with the Kurucz synthetic spectrum. The accretion luminosity is then derived transforming the R-band excess to a total excess by using a bolometric correction, which is adopted to be -0.4 mag. This is the value obtained by Hartigan et al. (1995) for an optically thick slab of hydrogen at $10^{4} \mathrm{~K}$, which is the typical temperature of the accretion shock (Sect. 4.1). However, it is noted that the bolometric correction is not based on a magnetospheric accretion model but on a boundary layer one. For this reason, we assume that the accuracy of the accretion rate estimated from this method is not better than \pm 1 dex. The resulting accretion luminosity, log $\left(\mathrm{L}_{\text {acc }} / \mathrm{L}_{\odot}\right) \sim 0.8$, can be transformed to a mass accretion rate from

$$
L_{\mathrm{acc}}=\frac{G M_{*} \dot{M}_{\mathrm{acc}}}{R_{*}},
$$

deriving $\dot{M}_{a c c} \sim 3 \times 10^{-7} \mathrm{M}_{\odot} \mathrm{yr}^{-1}$. 


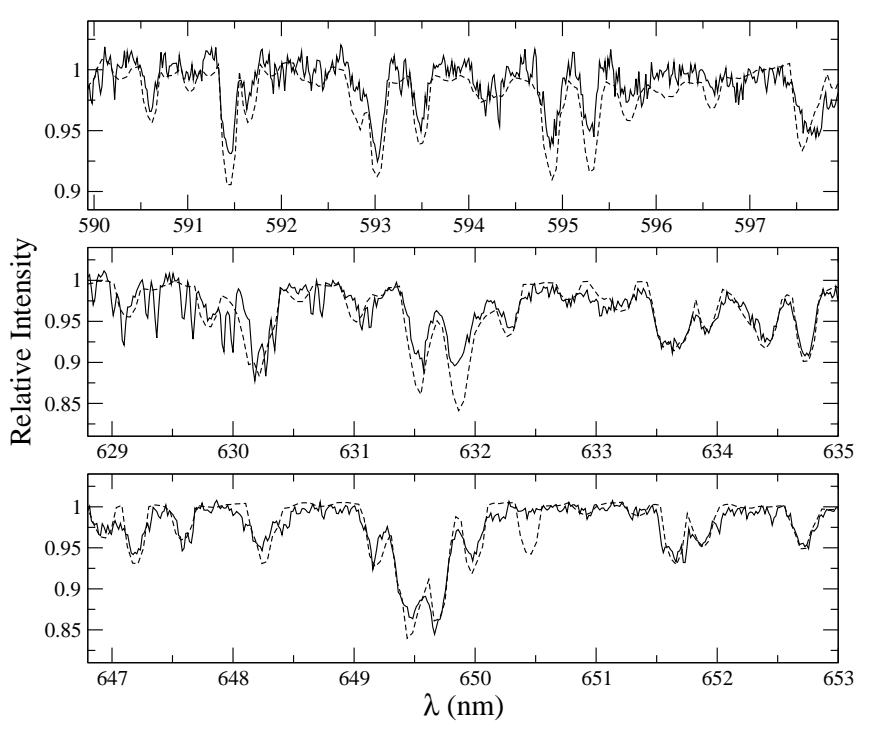

Figure 5. Several regions in the optical range of HD 142527 showing photospheric lines. The solid line is the observed spectrum whereas the dashed line is the broadened Kurucz spectrum representing the stellar photosphere.

\subsection{Line luminosities}

The accretion rate was also determined from the empirical correlations between the accretion luminosity and several emission lines that span from the UV to the nearIR region of the spectra. These have the form $\log \mathrm{L}_{a c c}$ $=\mathrm{a}+\mathrm{b} \times \log \mathrm{L}_{\text {line }}$, with $\mathrm{a}$ and $\mathrm{b}$ constants that depend on the specific line considered. Accretion luminosities are then transformed into mass accretion rates by using Eq. 3. Figure 6] shows the emission lines analyzed in this work, as observed in the X-Shooter spectrum of HD 142527. Table 2 lists their line equivalent widths and luminosities, the corresponding accretion luminosities and mass accretion rates, and the references from which the accretion-line luminosities correlations were obtained. Line luminosities were derived combining the circumstellar equivalent widths, obtained by subtracting the Kurucz photospheric spectrum from the observed one, and the continuum flux adjacent to each line, derived from the photometry indicated in Sect. 退. Line luminosities were dereddened considering the $\mathrm{E}(\mathrm{B}-\mathrm{V})$ value in Table 11, a standard value for the total to selective extinction ratio $\left(\mathrm{R}_{V}=3.1\right)$, and the extinction law in Cardelli et al. (1989). It is noted that the photospheric fluxes and the X-Shooter spectrum were taken on different dates, which could introduce some uncertainty. However, it is assumed that continuum variability is low for this object (Sect. 3). Therefore, it is not expected that results would significantly change if the photometry and the spectrum were taken simultaneously. In addition, the empirical correlations with the $\mathrm{Ca}$ IIK, $\mathrm{H} \gamma$, Na ID, and O I8446 lines were derived from stars with masses below the stellar mass of HD 142527, although we assume them as equally valid given that recent works indicate that these can be extrapolated to more massive, hotter regimes (Pogodin et al. 2012; Mendigutía et al. 2013).

According to these estimates, the mass accretion rate of HD 142527 is in between $\sim 6 \times 10^{-8}$ and $4 \times 10^{-7}$,

2 2MASS $\mathrm{JHK}_{s}$ photometry from the CDS catalogue II/246 (Cutri et al. 2003) was used for the lines in the NIR arm
Table 2

Line equivalent widths, luminosities, and accretion rates of HD 142527

\begin{tabular}{|c|c|c|c|c|c|}
\hline Line & $\begin{array}{l}\text { EW } \\
(\AA)\end{array}$ & $\begin{array}{r}\log \mathrm{L}_{\text {line }} \\
{\left[\mathrm{L}_{\odot}\right]}\end{array}$ & $\begin{array}{r}\log \mathrm{L}_{\text {acc }} \\
{\left[\mathrm{L}_{\odot}\right]}\end{array}$ & $\begin{array}{r}\log M_{a c c} \\
{\left[\mathrm{M}_{\odot} \mathrm{yr}^{-1}\right]}\end{array}$ & Ref \\
\hline Ca IIK (3934 Å) & -3.4 & -1.99 & $0.09 \pm 0.32$ & $-7.23 \pm 0.33$ & (1) \\
\hline $\mathrm{H} \gamma(4341 \AA)$ & -1.8 & 2.33 & $0.11 \pm 0.22$ & $-7.20 \pm 0.23$ & (1) \\
\hline $\mathrm{H} \beta(4861 \AA)$ & -3.8 & -2.01 & $0.44 \pm 0.21$ & $-6.88 \pm 0.22$ & (2) \\
\hline He I $(5876 \AA ̊)$ & -0.23 & -3.31 & $0.09 \pm 0.40$ & $-7.22 \pm 0.41$ & (3) \\
\hline Na ID $(5894 \AA)$ & -0.71 & -2.8 & $3 \pm 0.24$ & $-7.08 \pm 0.41$ & (1) \\
\hline $\mathrm{H} \alpha(6563 \AA)$ & -14 & -1.50 & $0.65 \pm 0.35$ & $-6.67 \pm 0.36$ & (4) \\
\hline O I $(8446 \AA)$ & -0.55 & -3.05 & $0.89 \pm 0.40$ & $-6.42 \pm 0.41$ & (1) \\
\hline Ca II $(8542 \AA)$ & -2.6 & -2.38 & $0.40 \pm 0.40$ & $-6.91 \pm 0.41$ & (3) \\
\hline $\operatorname{Pa} \gamma(10938 \AA)$ & -3.1 & -2.74 & $0.38 \pm 0.40$ & $-6.94 \pm 0.41$ & (5) \\
\hline $\mathrm{Pa} \beta(12818 \AA)$ & -6.5 & -2.42 & $0.39 \pm 0.40$ & $-6.93 \pm 0.41$ & (3) \\
\hline $\operatorname{Br} \gamma(21660 \AA)$ & -7.4 & -2.67 & $0.44 \pm 0.47$ & $-6.85 \pm 0.41$ & (6) \\
\hline
\end{tabular}

Notes. Typical errors for the circumstellar equivalent widths and dereddened line luminosities are $10 \%$ and $15 \%$, respectively. Error bars for the accretion luminosities consider those in the corresponding empirical calibrations, as indicated in the references provided in the last column. Error bars in the mass accretion rates consider those in the stellar parameters derived in this work. Data for $\mathrm{Na}$ ID $(5894 \AA)$ refers to the sum of both $\mathrm{D}_{1}$ and $\mathrm{D}_{2}$ components.

References. (1): Herczeg \& Hillenbrand (2008), (2): Fang et al. (2009), (3): Dahm (2008), (4): Mendigutía et al. (2011), (5): Gatti et al. (2008), (6): Calvet et al. (2004)

based on the line luminosity of $\mathrm{H} \gamma$ and $\mathrm{O}$ I8446, respectively. The mean accretion rate derived from the hydrogen recombination lines has been proposed to be the most accurate accretion rate estimate (Rigliaco et al. 2012), providing $1.3( \pm 0.5) \times 10^{-7} \mathrm{M} \odot \mathrm{yr}^{-1}$ (this uncertainty comes from the propagation of the ones for each line). However, the averaged value from the rest of the lines, $1.4( \pm 0.8) \times 10^{-7} \mathrm{M}_{\odot} \mathrm{yr}^{-1}$, is consistent within the uncertainties. Therefore, it is assumed that the best accretion rate estimate obtained through this method is the total average from all the emission lines, providing $1.4( \pm 0.4) \times 10^{-7} \mathrm{M}_{\odot} \mathrm{yr}^{-1}$.

\section{DISCUSSION AND CONCLUSSIONS}

We used a X-Shooter spectrum of HD 142527 to provide an accurate, independent determination of its stellar parameters and accretion rate. The stellar parameters are summarized in Table 1, the mass accretion rates obtained from the different tracers are plotted in Fig. 7 . All individual accretion rate estimates are consistent to each other, given that they lie within $\pm 3 \sigma$ from the mean value. Therefore, we consider this average value as the most robust estimate of the accretion rate of HD 142527: $2( \pm 1) \times 10^{-7} \mathrm{M}_{\odot} \mathrm{yr}^{-1}$, where the uncertainty is calculated from the propagation of the individual ones.

Garcia Lopez et al. (2006) estimated an accretion rate of $7 \times 10^{-8} \mathrm{M}_{\odot} \mathrm{yr}^{-1}$, assuming a distance of 200 pc and different stellar parameters than derived here. Therefore, this result cannot be directly compared to ours. In order to make an appropriate comparison, we first transformed the $\operatorname{Br} \gamma$ luminosity provided in Garcia Lopez et al. (2006) to the one that would be derived at a distance of $140 \mathrm{pc}$. The same $\mathrm{L}_{a c c}-\mathrm{L}_{B r \gamma}$ calibration was then applied, using the transformed line luminosity, to derive an accretion luminosity of $0.58 \mathrm{~L} \odot$, instead of the $1.01 \mathrm{~L}_{\odot}$ provided in that work. Finally, the corresponding mass accretion rate from Eq. 3. using the stellar parameters derived here, is $\sim 3 \times 10^{-8}$ 

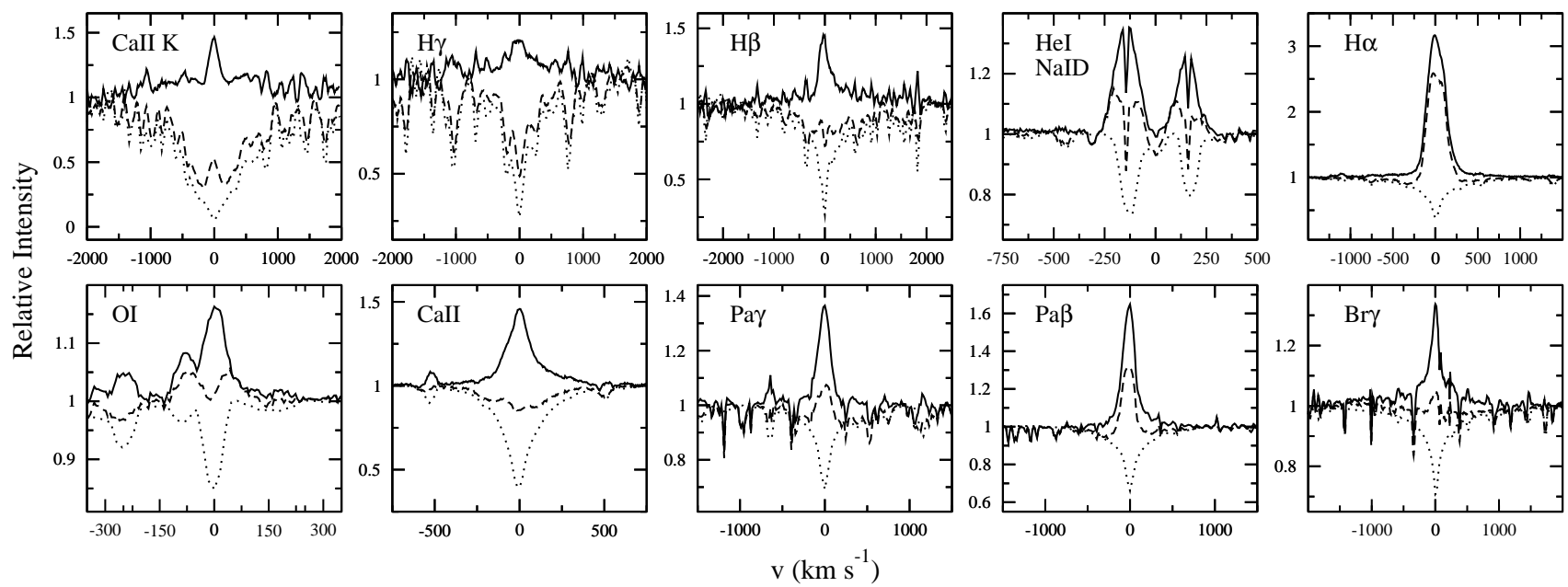

Figure 6. The spectral lines used as accretion tracers are shown in the different panels, ordered with increasing wavelength from left to right and from top to bottom. Dashed, dotted and solid lines represent the observed, photospheric (Kurucz) and circumstellar (observed photospheric) contributions, respectively.

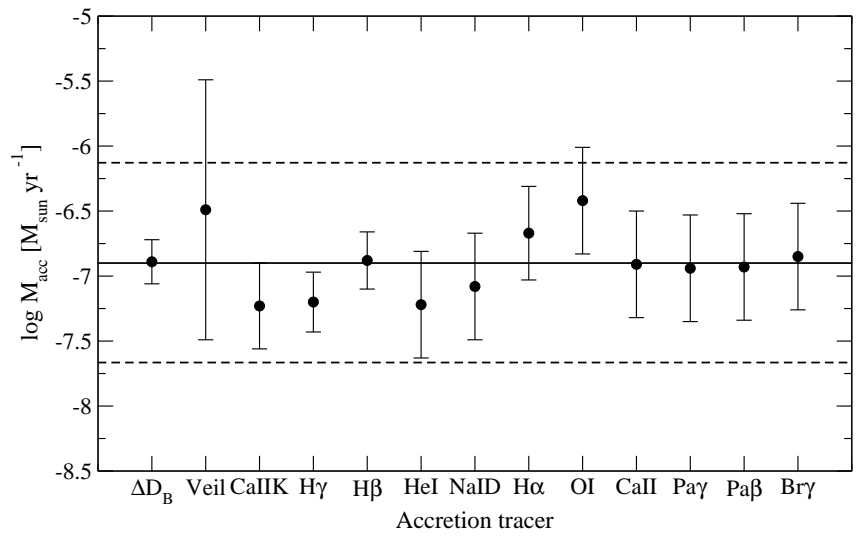

Figure 7. Accretion rates obtained for HD 142527 from the indicated accretion tracers in the x-axis. The horizontal solid line represents the average accretion rate and the dashed lines the \pm $3 \sigma$ deviation from the average.

$\mathrm{M}_{\odot} \mathrm{yr}^{-1}$, which is a factor $\sim 7$ lower than our estimate. The difference cannot be associated to different procedures but mainly to variability on a timescale of $5 \mathrm{yr}$, which is the time elapsed between the spectrum in Garcia Lopez et al. (2006) and ours. The Br $\gamma$ line in that work showed no emission whereas this can be observed in our X-Shooter spectrum (see Fig A.1 in Garcia Lopez et al. 2006, and our Fig. 6). Regarding the more recent accretion estimate from the Pfund $\beta$ line luminosity in Salyk et al. (2013, spectra taken on 2008), a mass accretion rate of $1 \times 10^{-7} \mathrm{M}_{\odot} \mathrm{yr}^{-1}$ was provided. Note that this value, clearly above the estimate in Garcia Lopez et al. (2006), refers to exactly the same stellar parameters and distance assumed in that work. However, that value translates again to $3 \times 10^{-8} \mathrm{M}_{\odot}$ $\mathrm{yr}^{-1}$, once the above described correction considering 140 pc and our stellar parameters is applyed. As mentioned by Salyk et al. (2013), their empirical calibration provides lower limit estimates for the accretion rate, given that the accretion measurements in Mendigutía et al. (2011) were not considered. If these measurements are taken into account, and using Fig. 8 from Salyk et al. (2013), a mass accretion rate consistent with our result is obtained. This would constrain the timescale of the observed variability of a factor $\sim 7$ in HD 142527 to only
2 yr.

Casassus et al. (2013) obtained a gas flow rate from the outer to the inner disk of HD 142527. According to that work, the gap-crossing filament they found transports a mass accretion rate in-between $7 \times 10^{-9}$ and 2 $\times 10^{-7} \mathrm{M}_{\odot} \mathrm{yr}^{-1}$. The lower limit was derived from the $\mathrm{HCO}^{+}$emission, assuming that the emitting gas is close to the critical density, $\mathrm{n}\left(\mathrm{H}_{2}\right) \sim 10^{6} \mathrm{~cm}^{-3}$. Because the gas is likely at a density higher than the critical density, $10^{-9} \mathrm{M}_{\odot} \mathrm{yr}^{-1}$ is a lower limit to the flow through the filament. The upper limit of this flow, $2 \times 10^{-7}$ $\mathrm{M}_{\odot} \mathrm{yr}^{-1}$, was derived from the dust continuum emission, assuming that the fraction of continnum emission from the filament is the same as the mass fraction of the filament relative to the total disk mass, and that the gas-to-dust ratio is uniform (and at the standard value) throughout the disk. However, this assumption may not be valid if the grain abundance in the filament differs from that of the disk at larger radii, which is the region responsible for most of the dust continuum emission. Rice et al. (2006) have argued that pressure gradients at the outer edge of a disk gap can act as a dust filter, letting particles smaller than a critical size (typically larger than $\sim 10 \mu \mathrm{m}$ ) through to the inner disc while holding back larger particles in the outer disc. As a result, this process can also produce a very large gasto-dust ratio in the filament, which would lead to an underestimate of its gas mass, and therefore of the accretion rate inferred by Casassus et al. (2013), potentially by a few orders of magnitude. Despite the uncertainty introduced by this possibility, it is unlikely that the true accretion rate through the filament would be much larger than the upper end of the accretion rate for $2 \mathrm{M}_{\odot}$ HAeBe stars $\left(\sim 10^{-6} \mathrm{M}_{\odot} \mathrm{yr}^{-1}\right.$; Donehew \& Brittain 2011; Mendigutía et al. 2011). Given the uncertainty on the gap flow, it is difficult to state whether it is larger, the same, or smaller than the stellar accretion rate derived in this work, and therefore what fraction, if any, could be intercepted by a possible planet. Nevertheless, the stellar accretion rate we have derived assuming the same distance of $140 \mathrm{pc}$ is consistent with the upper limit in Casassus et al. (2013). Under the hypothesis that the disk flow rate is constant -the X-Shooter spectrum used 
to provide our estimates was taken 2 yr before the observations analyzed in that work-, our stellar accretion rate would imply that almost all gas transferred from the outer to the inner disk is trapped by the central star, and only a small amount could be accreted onto the planet. This would be consistent with small planetary accretion rates, predicted to be in the range $\sim 10^{-9}-10^{-11} \mathrm{M}_{\odot}$ $\mathrm{yr}^{-1}$ (see e.g. Ayliffe \& Bate 2009; Machida et al. 2010). Time-monitoring of the simultaneous behaviour of the gas flow in the different parts of the HD 142527 system could provide interesting results on their dynamics and on the possible influence from a giant planet.

Finally, Biller et al. (2012) (see also Close et al. 2014) reported the detection of a low-mass stellar companion to HD142527 separated by 88 mas from the star and at a PA of 133 degrees, i.e., not co-located with the filament reported by Casassus et al. (2012). As reported in that work, the mass derived for the companion is strongly dependent on the precise age of the central object, assumed to be in the range 1-10 Myr. The tighter constraint that we find for the age of the system indicates that the mass of the possible companion should be in-between 0.20 and $0.35 \mathrm{M}_{\odot}$ (see Fig. 3 in Biller et al. 2012), based on the Baraffe et al. (1998) tracks.

In summary, accretion rate studies on objects with properties similar than HD 142527 are helpful not only to constrain accretion rate variability but also to give insight into possible stellar/planetary companions.

Based on observations made with ESO Telescopes at the La Silla Paranal Observatory under programme ID 084.C-0952. B. Montesinos is supported by Spanish grant AYA2011-26202. S.D. Brittain acknowledges support for this work from the National Science Foundation under grant number AST-0954811. The authors are grateful to Jesús Maíz-Apellániz for fruitful discussions concerning the photometry of this star. The authors thank the anonymous referee for his/her useful comments on the original manuscript, which helped us to improve the paper.

\section{Facilities: VLT:Kueyen}

\section{REFERENCES}

Ayliffe, B.A. \& Bate, M.R. 2009, MNRAS, 393, 49

Baines, D., Oudmaijer, R.D., Porter, J.M., Pozzo, M. 2006, MNRAS, 367,737

Baraffe, I., Chabrier, G., Allard, F., Hauschildt, P.H. 1998, A\&A, 337, 403

Biller, B., Lacour, S., Juhász, A. et al. 2012, ApJ, 753, L38

Bouvier, J., Alencar, S. H. P., Harries, T. J. et al. 2007, Protostars and Planets V, 479

Brittain, S.D., Najita, J.R., Carr, J.S. et al. 2013, ApJ, 767, 159

Calvet, N., \& Gullbring, E. 1998, ApJ, 509, 802

Calvet, N., Muzerolle, J., Briceño, C. et al. 2004, AJ, 128, 1294

Cardelli, J. A., Clayton, G. C., \& Mathis, J. S. 1989, ApJ, 345, 245

Casassus, S., Perez M.S., Jordán, A. et al. 2012, ApJ, 754, L31

Casassus, S., van der Plas, G., Perez, S. et al. 2013, Nature, 493, 191

Castelli, F., \& Kurucz, R.L., 2003, in IAU Symposium, Vol. 210, Modelling of Stellar Atmospheres, eds. N. Piskunov, W.W. Weiss, \& D.F. Gray, 20

Close, L.M., Follette, K.B., Males, J.R. et al. 2014, ApJL, 781, L30

Cutri, R.M., Skrutskie, M.F., Van Dyk, S., et al., 2003,

CDS/ADC Collection of Electronic Catalogues, 2246, 0 de Zeeuw, P.T., Hoogerwerf, R., de Bruijne, J.H.J., Brown, A.G.A., Blaauw, A. 1999, AJ, 117, 354

Dahm, S.E. 2008, AJ, 136, 521

Donehew, B., \& Brittain, S. 2011, AJ, 141, 46

Fang, M., van Boekel, R., Wang, W. et al. 2009, A\&A, 504, 461

Fressin, F., Torres, G., Charbonneau, D. et al. 2013, ApJ, 766, 81

Fujiwara, H., Honda, M., Kataza, H. et al. 2006, ApJ, 644, L133

Fukagawa, M., Tamura, M., Itoh, Y. et al. 2006, ApJ, 636, L153

Galli, P.A.B., Bertout, C., Teixeira, R., \& Ducourant, C., 2013 , A\&A, 558, 77

Garcia Lopez, R., Natta, A., Testi, L., Habart, E. 2006, A\&A, $459,837 \mathrm{G}$

Gatti, T., Natta, A., Randich, S., Testi, L., Sacco, G. 2008, A\&A, 481,423

Glebocki, R. \& Gnacinski, P., 2005, Catalog of Stellar Rotational Velocities, VizieR On-line Data Catalog: III/244

Gray, R.O. \& Corbally, C.J. 2009, Stellar Spectral Classification, Princeton University Press

Hartigan, P., Edwards, S., Ghandour, L. 1995, ApJ, 452, 736

Herczeg, G.J. \& Hillenbrand, L.A. 2008, ApJ, 681, 594

Houk, N. 1978, Michigan Catalogue of Two-Dimensional Spectral Types for the HD Stars, ed. N. Houk

Huélamo, N., Lacour, S., Tuthill, P. et al. 2011, A\&A, 528, L7

Koenigl, A. 1991, ApJ, 370, L39

Kraus, A.L. \& Ireland, M.J. 2012, ApJ, 745, 5

Lutz, T.E. \& Kelker, D.H., 1973, PASP, 85, 573

Machida, M.N., Kokubo, E.; Inutsuka, S.-I., Matsumoto, T. 2010, MNRAS, 405, 1227

Malfait, K., Bogaert, E., \& Waelkens, C., 1998, A\&A, 331, 211

Malfait, K., Waelkens, C., Bouwman, J., de Koter, A., Waters, L.B.F.M. 1999, A\&A, 345, 181

Manoj, P., Bhatt, H.C., Maheswar, G., Muneer, S. 2006, ApJ, 653,657

Mayor, M. \& Queloz, D. 1995, Nature, 378, 355

Mendigutía, I., Calvet, N., Montesinos, B. et al. 2011, A\&A, 535, A99

Mendigutía, I., Brittain, S., Eiroa, C. et al. 2013, ApJ, 776, 44

Modigliani, A., Goldoni, P., Royer, F., et al. 2010, in Society of Photo-Optical Instrumentation Engineers (SPIE) Conference Series, Vol. 7737

Montesinos, B., Eiroa, C., Mora, A., \& Merín, B. 2009, A\&A, 495,901

Mottram, J. C., Vink, J. S., Oudmaijer, R. D., \& Patel, M. 2007, MNRAS, 377, 1363

Munari, U., Sordo, R., Castelli, F., \& Zwitter, T. 2005, A\&A, 442,1127

Muzerolle, J., D'Alessio, P., Calvet, N., \& Hartmann, L. 2004, ApJ, 617, 406

Pogodin, M.A., Hubrig, S., Yudin, R.V. et al. 2012, AN, 333, 594

Rameau, J., Chauvin, G., Lagrange, A.M. et al. 2012, A\&A, 546, A24

Rice, W.K.M., Armitage, P.J., Wood, K., Lodato, G. 2006, MNRAS, 373, 1619

Rigliaco, E., Natta, A., Testi, L. et al. 2012, A\&A, 548, A56

Salyk, C.; Herczeg, G.J., Brown, J.M. et al. 2013, ApJ, 769, 21

Shu, F., Najita, J., Ostriker, E., et al. 1994, ApJ, 429, 781

Straizys, V., \& Kuriliene, G. 1981, Ap\&SS, 80, 353

Uchida, Y., \& Shibata, K. 1985, PASJ, 37, 515

van Boekel, R., Min, M., Waters, L.B.F.M. et al. 2005, A\&A, 437, 189

van den Ancker, M.E., De Winter, D., Tjin A Djie, H.R.E. 1998, A\&A, 330, 145

van Eyken, J.C., Ciardi, D.R., von Braun, K. et al. 2012, ApJ, 755,42

van Leeuwen, F., 2007, A\&A, 474, 653

Verhoeff, A.P., Min, M., Pantin, E. et al. 2011, A\&A, 528, A91

Vernet, J., Dekker, H., DOdorico, S. et al. 2011, A\&A, 536, A105

Vink, J. S., Drew, J. E., Harries, T. J., \& Oudmaijer, R. D. 2002, MNRAS, 337, 356

Waelkens, C., Waters, L.B.F.M., de Graauw, M.S. et al. 1996, A\&A, 315, L245

White, R.J., \& Basri, G. 2003, ApJ, 582, 1109

White, R.J., \& Hillenbrand, L.A. 2004, ApJ, 616, 998

Yi, S., Demarque, P., Kim, Y.-Ch., et al., 2001, ApJS, 136, 417 